\title{
Hard X-ray spectra and positions of solar flares observed by RHESSI: photospheric albedo, directivity and electron spectra
}

\author{
J. Kašparová ${ }^{1}$, E. P. Kontar ${ }^{2}$, and J. C. Brown ${ }^{2}$
}

\author{
1 Astronomical Institute, Academy of Sciences of the Czech Republic, Fričova 298, 25165 Ondřejov, Czech Republic \\ e-mail: kasparov@asu.cas.cz \\ 2 Department of Physics and Astronomy, University of Glasgow, G12 8QQ, UK \\ e-mail: eduard,john@astro.gla.ac.uk
}

Received 3 November 2006 / Accepted 31 January 2007

\begin{abstract}
Aims. We investigate the signature of the photospheric albedo contribution in solar flare hard X-ray spectra, the effect of low energy cutoffs in electron spectra, and the directivity of hard X-ray emission.

Methods. Using Ramaty High Energy Solar Spectroscopic Imager (RHESSI) flare data we perform a statistical analysis of spatially integrated spectra and positions of solar flares.

Results. We demonstrate clear centre-to-limb variation of photon spectral indices in the $15-20 \mathrm{keV}$ energy range and a weaker dependency in the $20-50 \mathrm{keV}$ range which is consistent with photospheric albedo as the cause. The results also suggest that low-energy cutoffs sometimes inferred in mean electron spectra are an artefact of albedo. We also derive the anisotropy (ratio of downward/observer directed photons) of hard X-ray emission in the $15-20 \mathrm{keV}$ range for various heliocentric angles.
\end{abstract}

Key words. Sun: flares - Sun: X-rays, gamma rays - Sun: particle emission - scattering - techniques: spectroscopic

\section{Introduction}

The successful operation of the Ramaty High Energy Solar Spectroscopic Imager RHESSI (Lin et al. 2002) since February 2002 has provided us with a substantial database of solar hard X-ray flares that can be used for detailed spectroscopic analysis. The high accuracy of the spectroscopic data, in combination with the positions of solar flares, allows us to determine the centre-to-limb spectral variations in various energy ranges.

Variation of hard X-ray flare occurrence and spectral index with solar position of the sources have been of great interest for several decades. As suggested e.g. by Elwert \& Haug (1971); Brown (1972); Petrosian (1973); Leach \& Petrosian (1983), centre-to-limb variations could provide essential information on directivity of X-ray emission and hence accelerated electrons. Most earlier analyses concentrated on centre-to-limb variation of flare occurrence. Ohki (1969) and Pintér (1969) reported significant centre-to-limb variation at energies above $10 \mathrm{keV}$ although they did not correct the hard X-ray flare occurrence for $\mathrm{H} \alpha$ flare longitude distribution. Subsequent works (Drake 1971; Catalano \& van Allen 1973; Phillips 1973; Datlowe et al. 1974; Kane 1974) covering various energy ranges from $1-10 \mathrm{keV}$ claimed no centre-to-limb variation of hard X-ray occurrence. Datlowe et al. (1977) concluded that anisotropy in the $10-100 \mathrm{keV}$ range was so low that it ruled out the downward-beamed thick target model due to lack of limb brightening predicted e.g. by (Brown 1972). Theoretical models (Petrosian 1973; Langer \& Petrosian 1977; Bai \& Ramaty 1978) predicted that at hard X-ray energies, above $15 \mathrm{keV}$, centre-to-limb variations of spectral properties should be sensitive to electron directivity. Although such studies were not very common, Datlowe et al. (1974) and Roy \& Datlowe (1975) found, contrary to above mentioned works based on hard $\mathrm{X}$-ray occurrence, significant centre-to-limb spectral variation in the $10-100 \mathrm{keV}$ range and attributed it to anisotropy of X-ray emission. Then, Vestrand et al. (1987) demonstrated that Solar Maximum Mission (SMM) GRS data in the 25-200 keV range and above $300 \mathrm{keV}$, show significant spectral hardening of events near the limb. Similar results were reported by Bogovalov et al. (1985) from Venera 13 data in the $50-100 \mathrm{keV}$ range and by McTiernan \& Petrosian (1991) in the 0.3-1 MeV range.

It has been known for a while that X-ray photons can be effectively backscattered by photosphere atoms and electrons (Tomblin 1972; Bai \& Ramaty 1978). Thus, at energies not dominated by absorption the backscattered albedo flux must be seen virtually in every solar flare spectrum, the degree of the albedo contribution depending on the directivity of the primary X-ray flux (Kontar et al. 2006). The solar flare photons backscattered by the solar photosphere can contribute significantly (the reflected flux is $50-90 \%$ of the primary in the $30-50 \mathrm{keV}$ range for isotropic sources) to the total observed photon spectrum. For the simple case of a power-law-like primary solar flare spectrum (without albedo), the photons reflected by the photosphere produce a broad "hump" component. Photospheric albedo makes the observed spectrum flatter below $\sim 35 \mathrm{keV}$ and slightly steeper above, in comparison with the primary spectrum. The amount of backscattered photons is given by the scattering cross-section and is roughly dependent on the projected area of the albedo patch, which makes the photospheric albedo stronger for solar centre events. Photospheric albedo has been treated using Monte Carlo simulations to calculate the observed spectrum for an assumed primary power-law spectrum (Bai \& Ramaty 1978). Kontar et al. (2006) developed a new approach to photospheric albedo based on the Green's functions of Magdziarz \& Zdziarski (1995) that produces the correction for any solar flare $\mathrm{X}$-ray spectrum and can be used both for forward and inverse spectrum calculations. The influence of albedo was not taken 
into account for spectral centre-to-limb variation in earlier analyses and it was wrongly dismissed (Datlowe et al. 1974) as being unimportant in the 20-50 keV range. However, Bai \& Ramaty (1978) and Vestrand et al. (1987) pointed out that the Datlowe's results are consistent with an albedo contribution to the observed photon spectrum. Using the Bai \& Ramaty (1978) results, the importance of albedo in electron spectrum inference was first included by Johns \& Lin (1992) and analysed by Alexander \& Brown (2002).

On the other hand, it has been long believed that the mean electron flux distribution $\bar{F}(E)$ defined by (Brown 1971; Brown et al. 2003) can exhibit low-energy cutoff - e.g. Nitta et al. (1990) showed that photon spectra of the impulsive component flatten toward low energies and suggested that a low-energy cutoff of the electron spectrum around $50 \mathrm{keV}$ could be responsible. Fárník et al. (1997), using Yohkoh data, observed a flare with a flat spectrum below $33 \mathrm{keV}$ (photon spectral index as low as 1.98). More recently, Kašparová et al. (2005) reported a clear low-energy cutoff inferred from the observed photon spectra of the August 20, 2002 flare, neglecting albedo. Using a regularised inversion technique, Kontar \& Brown (2006a) report three flares with a similar feature - an absence of electrons around 20-30 keV between possibly thermal and non-thermal component.

However, applying the model-independent albedo correction method of Kontar et al. (2006) to the August 20, 2002 flare, Kašparová et al. (2005) have clearly demonstrated that such a high value of low-energy cutoff is removed by albedo correction for an isotropic source. Equally, the albedo corrected photon spectra do not require a gap in the inverted electron spectrum (Kašparová et al. 2005; Kontar \& Brown 2006a). Using the present survey, we can potentially find many flares with such low spectral indices that they might have clear low-energy cutoff appearing as a gap between thermal and non-thermal distributions.

The spectrum-independent photospheric albedo correction method is introduced in Sect. 2. Data selection and analysis are discussed in Sect. 3. In Sect. 4, our survey shows that the centreto-limb variations of spectral indices are consistent with photospheric albedo. Moreover, we find limits on the X-ray spectral directivity for various heliospheric angles. Section 5 confirms the prediction that the low-energy cutoff in $\bar{F}(E)$ is removed when the spectra are albedo corrected for all events with low spectral index found in the survey. Lastly, Sect. 6 reviews the results obtained and their consequences for the angular distribution of X-ray emitting electrons.

\section{Photospheric albedo and spectral index}

The albedo contribution produces substantial change to the observed spectrum in the range between 10 and $100 \mathrm{keV}$. It becomes negligible for energies below $10 \mathrm{keV}$ due to the photoelectric absorption by atoms while photons above $100 \mathrm{keV}$ penetrate deep into high density layers of the solar atmosphere and do not escape back. Therefore, the reflectivity of the photosphere has a broad maximum in the $30-50 \mathrm{keV}$ range. The total spectrum in the observer direction $\mu$ ( $\mu=\cos \theta$, where $\theta$ is the heliocentric angle) can be expressed as

$$
\begin{aligned}
& I_{\mathrm{o}}(\epsilon, \mu)=I_{\mathrm{p}}(\epsilon, \mu)+I_{\mathrm{A}}(\epsilon, \mu) \\
& I_{\mathrm{A}}(\epsilon, \mu)=\mathbf{G}\left(\epsilon, \epsilon^{\prime}, \mu\right) I_{\mathrm{p}}\left(\epsilon^{\prime}, \mu\right) \alpha(\mu),
\end{aligned}
$$

where $I_{\mathrm{p}}(\epsilon, \mu)$ is the primary photon spectrum of the flare, $I_{\mathrm{A}}(\epsilon, \mu)$ is the spectrum reflected into the observer direction, $\mathbf{G}$ is
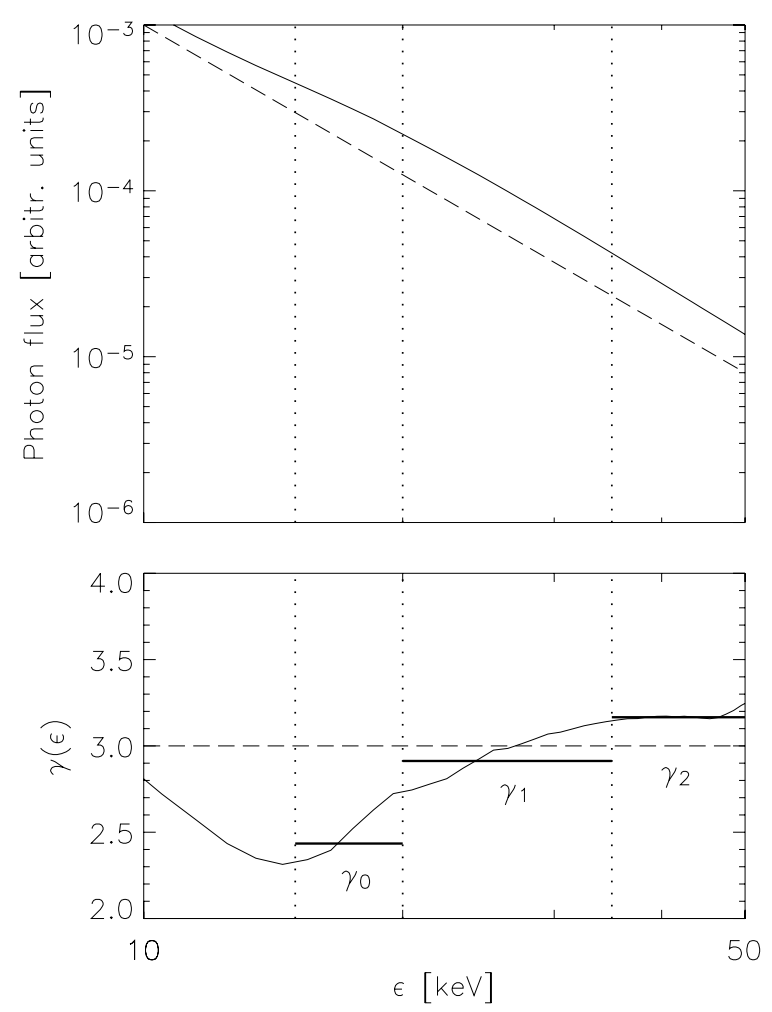

Fig. 1. Photon spectrum $I(\epsilon)$ (upper panel) and spectrum index $\gamma(\epsilon)$ (lower panel) for a modelled primary photon spectrum $I_{\mathrm{p}}(\epsilon) \sim \epsilon^{-3}$ for an isotropic source located at $\mu=0.8$. Primary $I_{\mathrm{p}}(\epsilon)$ and $\gamma_{\mathrm{p}}(\epsilon)$ are plotted with a dashed line and the observed $I_{0}(\epsilon)$ and $\gamma_{\mathrm{o}}(\epsilon)$ with a solid line. Horizontal lines indicate spectral indices $\gamma_{0}, \gamma_{1}$, and $\gamma_{2}$ obtained by single power-law fits in the $15-20,20-35$, and $35-50 \mathrm{keV}$ energy ranges, respectively.

the Green's matrix (Kontar et al. 2006) accounting for Compton backscattering and photoelectric absorption, and $\alpha(\mu)$ is a parameter of emission anisotropy, roughly the ratio of the flux toward the Sun $I_{\mathrm{p}}(\epsilon, \mu<0)=\alpha(\mu) I_{\mathrm{p}}(\epsilon, \mu)$ and the flux toward the observer $I_{\mathrm{p}}(\epsilon, \mu)$. We simplify the problem here by considering only $\alpha(\mu)$ independent of $\epsilon$ (cf. Alexander \& Brown 2006).

The photon spectrum, $I_{\mathrm{A}}(\epsilon, \mu)$, backscattered toward the observer strongly depends on three parameters: the spectral index of the primary spectrum, the location of the flare on the solar disc, and the directivity of X-ray emission (anisotropy parameter $\alpha(\mu)$ ). The contribution of the albedo to the observed spectrum is greater for flatter primary spectra (low $\gamma$ ) and for solar disc flares (large $\mu$ ). Further, the more X-rays are beamed downwards $\alpha(\mu)>1$, the larger is the contribution of the albedo to the observed spectrum.

The energy dependent spectral index

$\gamma(\epsilon) \equiv-\frac{\epsilon}{I(\epsilon)} \frac{\mathrm{d} I(\epsilon)}{\mathrm{d} \epsilon}=-\frac{\mathrm{d} \ln (I(\epsilon))}{\mathrm{d} \ln (\epsilon)}$

for the primary spectrum $\gamma_{\mathrm{p}}(\epsilon)$ substantially differs from that $\left(\gamma_{\mathrm{o}}(\epsilon)\right)$ of the total $I_{\mathrm{o}}(\epsilon, \mu)$ toward the observer (including albedo) - see Fig. 1 for the case of $\gamma_{\mathrm{p}}=3$. In the range $10-25 \mathrm{keV}$ the photon spectrum becomes flatter, $\gamma_{\mathrm{o}}<\gamma_{\mathrm{p}}$, while the observed spectral index is higher at energies above $35 \mathrm{keV}$, $\gamma_{\mathrm{o}}>\gamma_{\mathrm{p}}$.

Using a single power-law fit in various energy ranges (Fig. 1), we can estimate the local spectral index assuming it is constant in each energy range. (Note that generally for a photon 
spectrum $I(\epsilon) \sim \epsilon^{-c(\epsilon)}, c(\epsilon)=\gamma(\epsilon)$ only for $c=$ const (Conway et al. 2003).)

Hence, as a result of heliocentric angle dependent albedo, the spectral shape of photon spectra should vary as a function of their position on the solar disc, i.e. a centre-to-limb variation of spectral indices is expected and the degree of the spectral index variation with heliocentric angle could usefully provide us with the directivity of hard X-ray sources, i.e. the anisotropy coefficient $\alpha(\mu)$.

\section{Data selection and analysis}

In our analysis we used the whole RHESSI flare list (from February 12, 2002 until April 18, 2006) to search for any evidence of centre-to-limb variation in photon spectra.

RHESSI has 9 detectors on board with FWHM resolution about $\sim 1 \mathrm{keV}$. For X-ray spectroscopy analysis we use the six front segments of the detectors ignoring detector 2, 5 and 7 due to either low resolution or the segmentation problem (Smith et al. 2002).

To handle such a large set of data, we set up an automatic procedure which selects suitable events and retrieves the flare photon spectra. To be chosen for further analysis the events had to meet the following major criteria:

- The spectra of the events should be observed above $25 \mathrm{keV}$. Thus, we have selected flares flagged as observed above that energy from the RHESSI flare list and have determined the time of peak flux in the $25-50 \mathrm{keV}$ range. The events flagged as particle events were ignored. This led us to $\sim 1500$ events.

- Count rates exceeding about $2 \times 10^{3}$ counts per second per detector cause pulse pile-up in the detectors that requires troublesome correction (Smith et al. 2002). To avoid the pile-up issues, we have thrown away events with corrected livetime counter $<90 \%$. We disregarded also weak events (background subtracted count rate lower than 5 counts per second in the $25-50 \mathrm{keV}$ range) and events possibly affected by X-ray absorption in Earth's atmosphere (peak time closer than $60 \mathrm{~s}$ to RHESSI eclipse time). This reduces our survey to $\sim 800$ events.

- We have used CLEANed images in the range 10-20 and 20-50 keV to check for true solar events and to find their positions. Finally, the total number of the selected flare events drops to 703 .

RHESSI is an instrument with high background that makes background subtraction an important step in the data analysis (Schwartz et al. 2002). The code searches for two time intervals, one before and one after the flare peak, where count rates are less than 0.01 of the peak count rate in $15-50 \mathrm{keV}$ range and 0.1 above $50 \mathrm{keV}$. The background time intervals are determined for several energy bands separately and the background at the peak is estimated by a linear fit (Schwartz et al. 2002). See the flare example in Fig. 2.

The count spectra [counts $\mathrm{s}^{-1} \mathrm{keV}^{-1} \mathrm{~cm}^{-2}$ ] were accumulated in $12 \mathrm{~s}$ time intervals with a quasi-logarithmic energy binning from 10 to $100 \mathrm{keV}$ to improve the signal-to-noise ratio $(S N R)$ at higher energies (Fig. 2). After background subtraction, the resulting peak count flux (one spectrum per flare) and the detector response matrix (DRM) for the instrument at this time interval were used as an input for the regularised inversion routine (Scullion et al. in preparation; Kontar et al. 2004). The regularised inversion of a count flux spectrum gave us a nonparametric photon spectrum and its uncertainties.
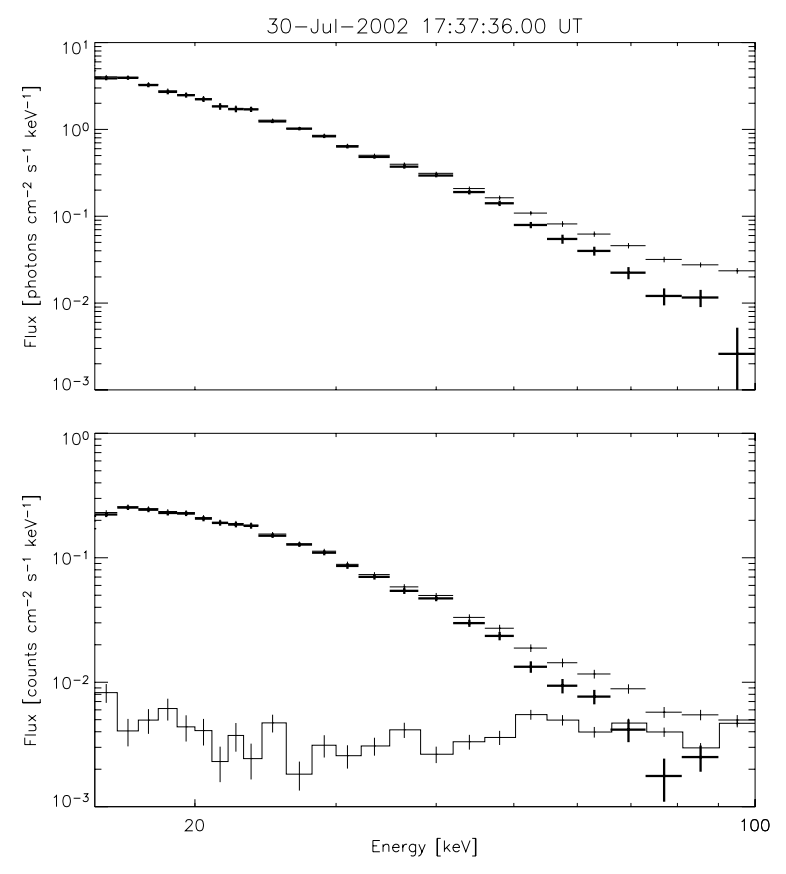

Fig. 2. Example of the flare spectrum for July 30, 2002 flare, 17:37:36-17:37:48 UT. The upper panel corresponds to the regularised photon spectrum, the lower panel shows the count spectrum. Observed spectrum is plotted with thin crosses, background subtracted flare spectrum with thick crosses. The background is displayed as a histogram. Horizontal sizes of crosses and width of the histogram bins correspond to widths of energy bands, while vertical sizes represent $1 \sigma$ statistical uncertainties of the flux.

\subsection{Photon spectra of selected events}

As discussed in Sect. 2, addition of the albedo contribution changes the shape of the primary spectra. To describe its effects, the regularised photon spectra have been fitted with a single power-law in three separate energy ranges 15-20, 20-35, and 35-50 keV thus obtaining corresponding spectral indices $\gamma_{0}, \gamma_{1}$, and $\gamma_{2}$, respectively.

Note that spectra in the $15-20 \mathrm{keV}$ energy range may be affected by thermal component(s) which could be comparable to or dominate the non-thermal emission and masking the albedo contribution. Considering a widely used isothermal approximation, value of $\gamma_{0}$ in such flares would be higher in comparison with events without strong thermal component.

Since the count flux decreases with energy while the background level does not (Fig. 2), naturally not all 703 events have significant flux above background at all three energy ranges. For $\gamma_{0}$ and $\gamma_{1}$ analysis we accepted only those events with count flux signal-to-noise ratio $(S N R)>3$ at all three energy ranges. Furthermore, the Green's matrices and therefore the corrected DRM are non-diagonal at $\gamma_{2}$ energies, the photon flux at this energy range depending on the signal at higher energies and making $\gamma_{2}$ unreliable for flares with low $S N R$ above $50 \mathrm{keV}$. Thus, for $\gamma_{2}$ analysis additional condition $S N R>3$ in the $50-66 \mathrm{keV}$ energy range was required.

Hence, the above conditions select only events with significant flux above the roughly estimated background in energies higher than the energy range where the spectral indices are determined. This reduced our set to 398 events for $\gamma_{0}$ and $\gamma_{1}$ analysis and to 123 events for $\gamma_{2}$ analysis - see Fig. 3. Note that the events are not uniformly distributed over $\mu$. The main reason is that active regions and flares are located in a rather small range 

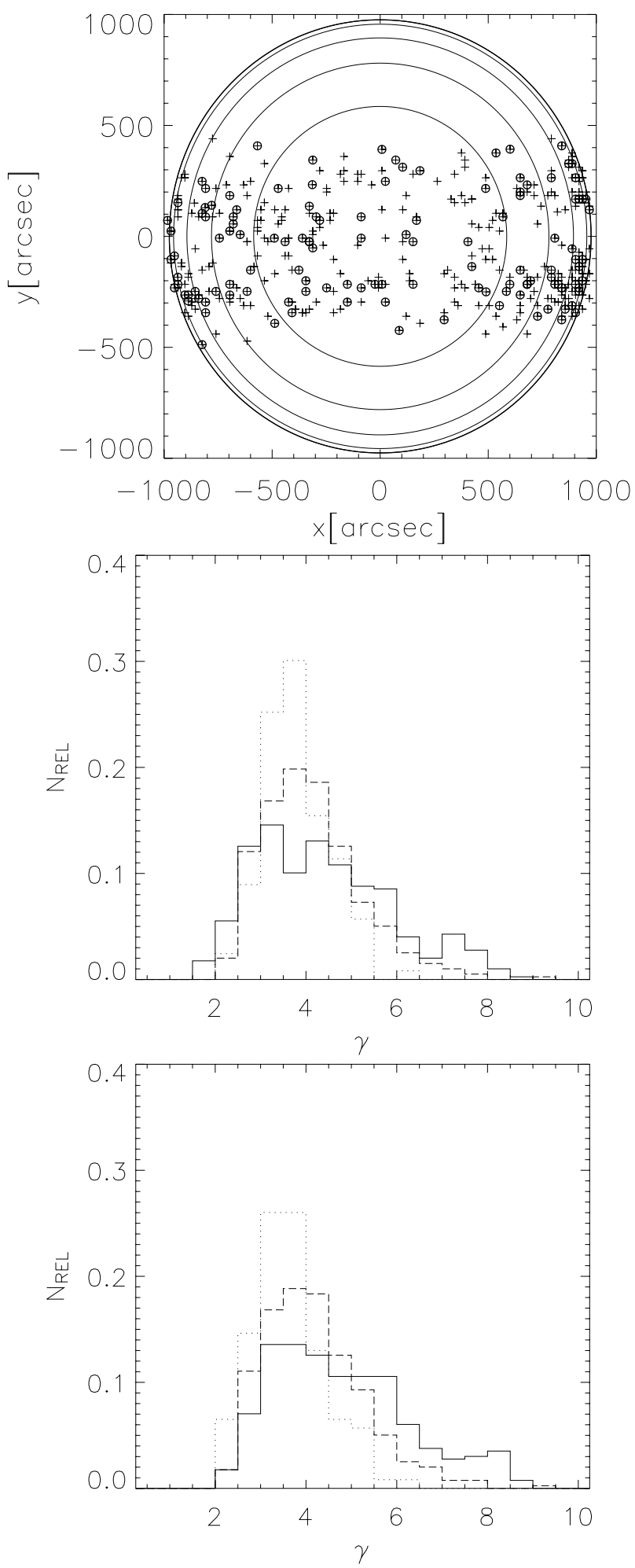

Fig. 3. Top: positions of selected flares. Crosses indicate 398 flares used for the centre-to-limb analysis of $\gamma_{0}$ and $\gamma_{1}$, small circles correspond to 123 flares for the $\gamma_{2}$ analysis (see text for details). Large circles indicate regions of $\mu=0.2,0.4,0.6$, and 0.8 . Middle and bottom: relative occurrence of $\gamma_{0}$ (solid), $\gamma_{1}$ (dashed), and $\gamma_{2}$ (dotted) from observed spectra and those corrected for isotropic albedo, respectively.

of latitudes. Thus fewer events per unit $\mu$ are observed close to the limb than at the disc centre. This also puts a constraint on the width of $\mu$ bins for anisotropy analysis - see Sect. 4.1 and Table 2.
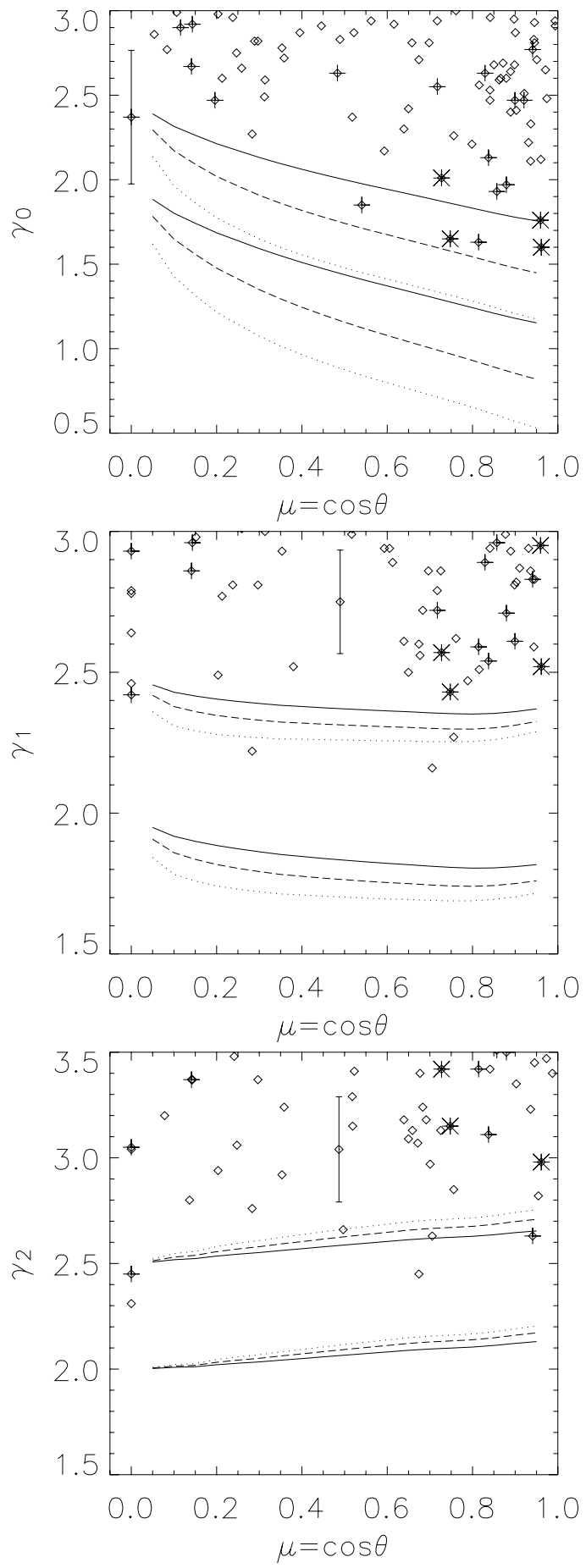

Fig. 4. Spectral indices $\gamma_{0}, \gamma_{1}$, and $\gamma_{2}$ versus cosine $\mu$ of heliocentric angle $\theta$. Vertical error bars indicate average uncertainties on the values as determined from single power-law fits. Lines show the predicted dependency for single power-law primary spectra with $\gamma_{\mathrm{p}}=2.0,2.5$ and for $\alpha(\mu)=1,2,4$ (solid, dashed, and dotted lines respectively), see also Sect. 4.1. High values of spectral indices are not shown to emphasize their lower bounds. Crosses represent flares for which $\bar{F}(E)$ was determined from the total spectra. Flares with a dip in $\bar{F}(E)$ are denoted as stars (see Sect. 5).

We also apply an isotropic $(\alpha(\mu)=1)$ albedo correction using Eq. (1) and the approach discussed in Kontar et al. (2006) to determine spectral indices of albedo corrected spectra. The relative occurrence of spectral indices of observed spectra and of those corrected for isotropic albedo is shown in Fig. 3. Note 
Table 1. Mean spectral indices $\overline{\gamma_{0}}, \overline{\gamma_{1}}$ and $\overline{\gamma_{2}}$ for all selected events at selected ranges of $\mu$. K-S prob. corresponds to probability that a pair of distributions comes from the same parent distribution. The number of events in each $\mu$ bin is indicated in brackets.

\begin{tabular}{lcccc}
\hline \hline albedo corr. & $\mu$ & $\bar{\gamma}_{0}(\mathrm{~N})$ & $\bar{\gamma}_{1}(\mathrm{~N})$ & $\bar{\gamma}_{2}(\mathrm{~N})$ \\
\hline none & $\mu<0.5$ & $4.6 \pm 0.1(157)$ & $4.2 \pm 0.1(157)$ & $3.61 \pm 0.09(45)$ \\
none & $\mu \geq 0.5$ & $4.3 \pm 0.1(241)$ & $4.07 \pm 0.06(241)$ & $3.88 \pm 0.09(78)$ \\
\hline K-S prob. & & 0.2 & 0.2 & 0.2 \\
\hline$\alpha(\mu)=1$ & $\mu<0.5$ & $4.8 \pm 0.1(157)$ & $4.3 \pm 0.1(157)$ & $3.5 \pm 0.1(45)$ \\
$\alpha(\mu)=1$ & $\mu \geq 0.5$ & $4.8 \pm 0.1(241)$ & $4.12 \pm 0.06(241)$ & $3.6 \pm 0.1(78)$ \\
\hline K-S prob. & & 1.0 & 0.2 & 0.5 \\
\hline none & $\mu<0.1$ & $4.7 \pm 0.2(49)$ & $4.4 \pm 0.2(49)$ & $3.5 \pm 0.2(13)$ \\
none & $\mu>0.9$ & $4.2 \pm 0.2(81)$ & $4.1 \pm 0.1(81)$ & $3.9 \pm 0.1(29)$ \\
\hline K-S prob. & & 0.009 & 0.4 & 0.4 \\
\hline$\alpha(\mu)=1$ & $\mu<0.1$ & $4.8 \pm 0.2(49)$ & $4.4 \pm 0.2(49)$ & $3.4 \pm 0.2(13)$ \\
$\alpha(\mu)=1$ & $\mu>0.9$ & $4.8 \pm 0.2(81)$ & $4.2 \pm 0.1(81)$ & $3.6 \pm 0.1(29)$ \\
\hline K-S prob. & \multicolumn{5}{c}{0.4} & 0.4 & 0.8 \\
\hline
\end{tabular}

the excess of events with $\gamma_{0}<2$ which is removed after the isotropic albedo correction. The excess of $\gamma_{0}>7$ corresponds to events with very steep spectra.

\section{Centre-to-limb variation}

The resulting spectral indices $\gamma_{0}, \gamma_{1}$, and $\gamma_{2}$ of the flares are shown Fig. 4. There is no clear upper bound on $\gamma$ values (primary and observed spectral index can be arbitrarily large) and the albedo contribution to the observed spectrum becomes negligible for steep primary spectrum - see Sect. 2. On the contrary, the primary spectral index for bremsstrahlung emission cannot be lower than 1 . Therefore, for display purposes only values up to 3 (3.5 for $\gamma_{2}$ ) are shown; events with larger spectral indices fill the whole range of $\mu$.

The spectral index $\gamma_{0}$ shows significant centre-to-limb variation (see Fig. 4). Lower values of $\gamma_{0}$ tend to be located closer to disc centre, forming an edge in the $\gamma_{0}(\mu)$ distribution. There are no flares with $\gamma_{0}<2$ located at $\mu<0.5\left(\theta>60^{\circ}\right)$, all such being at $\mu \geq 0.5$. We do not see similar pattern at higher energies (spectral indices $\gamma_{1}$ and $\gamma_{2}$ ) and the Fig. 4 shows no correlation with the position of flares in these energy ranges.

As one can see from our albedo model with $\alpha \neq \alpha(\epsilon)$ (Fig. 1), the strongest centre-to-limb variation due to photospheric albedo is expected at lower energies, i.e. for $\gamma_{0}$, and almost no dependency is expected on flare position at higher energies. The corresponding theoretical model for single power-law primary spectra is shown by various lines in Fig. 4.

Figure 4 also shows that anisotropy plays a significant role in centre-to-limb spectral variation only at $\gamma_{0}$ energies. Although we have no information about the primary spectra and distribution of primary spectral indices of analysed events, it is clear from the figure that the albedo model predictions, assuming single power-law primary spectra, are consistent with the general variations of the observed spectral indices with $\mu$. In order to put further constraint on the model, particularly at energies above $20 \mathrm{keV}$, spectral indices need to be determined with uncertainties smaller than the predicted centre-to-limb variation - see Fig. 4.

Following previous results (Vestrand et al. 1987; Datlowe et al. 1974, 1977), and to assess the change of spectral indices with $\mu$, we divide the sample into two subsets; $\mu<0.5$, and $\mu \geq 0.5$ and for each interval we calculate the mean values of $\gamma_{0}, \gamma_{1}$, and $\gamma_{2}$ of observed and isotropic albedo corrected photon spectra. We then apply the Kolmogorov-Smirnov (K-S) test (Press et al. 1992) to calculate the probability that the distributions of spectral indices at $\mu<0.5$ and $\mu \geq 0.5$ are drawn from the same parent distribution. The mean values and the probabilities (see Table 1) show the trend expected for isotropic albedo. The mean $\overline{\gamma_{0}}$ value of $\gamma_{0}$ increases after the albedo correction is applied as one would expect. The mean spectral indices $\overline{\gamma_{1}}, \overline{\gamma_{2}}$ with and without albedo correction do not differ significantly - i.e. are within the uncertainties of the mean values. The K-S probabilities show a similar trend, the distributions with the albedo component removed show larger probabilities of being drawn from the same dataset. However, the probability values for observed indices are too large to reject the null hypothesis, partly because the albedo contribution is smeared over too broad a range of $\mu, \Delta \mu=0.5$.

Better results can be achieved by taking two more extreme distinct sets of flares; one close to the limb with $\mu<0.1(\theta>$ $84^{\circ}$ ), where the albedo is negligible, and disc centre events with $\mu \geq 0.9\left(\theta \leq 25^{\circ}\right)$ where the albedo should be strongest. The value of K-S probability for the $\gamma_{0}$ distributions drops to $0.9 \%$, allowing us to reject the null hypothesis at 0.05 and 0.01 significant levels and conclude that the $\gamma_{0}$ distributions are "significantly different".

After applying the isotropic albedo correction, the $\gamma_{0}$ distributions can no longer be considered as being different - see Table 1 and the $\gamma_{0}$ distributions in Fig. 5. However, the same conclusion cannot be reached for $\gamma_{1}$ and $\gamma_{2}-$ see Table 1. It should be pointed out that the number of events for $\gamma_{2}$ analysis for those $\mu$ bins is rather low - see Table 1 - so a larger set of events will be needed to reach any conclusion.

\subsection{Directivity of $X$-ray emission}

The intensity of photons backscattered from the photosphere is determined by the downward directed primary photon spectrum. The larger the downward directivity of the source, the stronger is the albedo contribution to the observed spectrum (see the predictions for $\alpha=1,2,4$ and the model of a single power-law primary spectrum in Fig. 4). We describe the downward versus toward observer flux ratio by the anisotropy parameter $\alpha(\mu)$, see Eq. (1) (which we take to be independent of energy).

In order to assess the range of primary X-ray directivity consistent with the data, we have assumed limb spectra, $I_{0}(\epsilon, \mu<$ $0.1)$, to be true primary spectra toward the observer, $I_{\mathrm{p}}(\epsilon, \mu) \equiv$ $I_{\mathrm{o}}(\epsilon, \mu<0.1)$, and added to these the albedo component for various $\alpha(\mu)$. Using Eq. (1), the modelled spectra $I_{\mathrm{M}}$ in the observer direction $\mu$ take the form

$I_{\mathrm{M}}(\epsilon, \mu)=\left[\mathbf{1}+\alpha(\mu) \mathbf{G}\left(\epsilon, \epsilon^{\prime}, \mu\right)\right] I_{\mathrm{o}}\left(\epsilon^{\prime}, \mu<0.1\right)$.

This approach does not decrease the signal-to-noise ratio of photon flux as in the case of albedo subtraction which reduces the 
Table 2. K-S prob. for several values of directivities at different ranges of $\mu$ ( $N$ is the number of events in that range). The values correspond to probability that the observed distribution of $\gamma_{0}$ at a given range of $\mu$ and the modelled distribution $I_{\mathrm{M}}$ of $\gamma_{0}$ determined from the events at the limb $(\mu<0.1)$ with added albedo contribution for the given $\mu$ and directivity $\alpha$ are drawn from the same parent distribution.

\begin{tabular}{cccccccccc}
\hline \hline & & \multicolumn{7}{c}{ K-S prob. for $\alpha(\mu)$} \\
\cline { 3 - 10 }$\mu$ & $N$ & 10 & 5 & 4 & 2 & 1 & 0.5 & 0.25 & 0.2 \\
\hline $0.1-0.4$ & 80 & $3 \times 10^{-3}$ & 0.03 & 0.07 & 0.2 & 0.07 & 0.02 & $8 \times 10^{-3}$ & $6 \times 10^{-3}$ \\
$0.4-0.7$ & 88 & $9 \times 10^{-6}$ & $4 \times 10^{-4}$ & $1 \times 10^{-3}$ & 0.02 & 0.4 & 0.1 & 0.03 & 0.02 \\
$0.7-0.9$ & 100 & $7 \times 10^{-6}$ & $2 \times 10^{-4}$ & $4 \times 10^{-4}$ & 0.02 & 0.3 & 0.04 & $7 \times 10^{-3}$ & $7 \times 10^{-3}$ \\
$0.9-1.0$ & 81 & $2 \times 10^{-3}$ & $9 \times 10^{-3}$ & 0.04 & 0.2 & 0.5 & 0.06 & 0.01 & $4 \times 10^{-3}$ \\
\hline
\end{tabular}

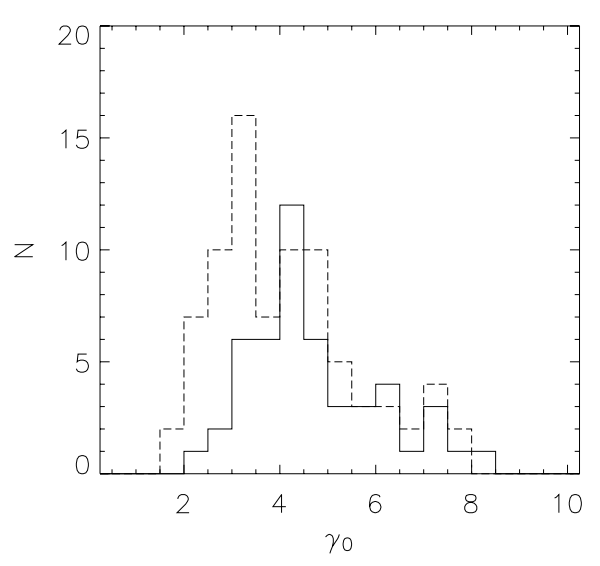

photon flux but keeps the corresponding uncertainty at the same level (uncertainty on the albedo contribution is set to zero.).

The K-S test was then applied to distributions of spectral indices of the modelled $I_{\mathrm{M}}$ and observed $I_{\mathrm{o}}$ spectra in several ranges of $\mu$. The width of the $\mu$ bins was chosen to contain approximately the same number of events. Due to the non-uniform distribution of events with $\mu$, see Fig. 3, the width of the $\mu$ bins increases towards the limb (smaller $\mu$ ). We regard the directivity $\alpha(\mu)$ as consistent with the data if the $\mathrm{K}-\mathrm{S}$ probability is larger than 0.05 or 0.01 significance level, i.e. when the null hypothesis that two distributions of observed and modelled spectral index for given $\alpha(\mu)$ are drawn from the same distribution cannot be rejected. In the $\gamma_{0}$ energy range we find the ranges of allowable directivity to be $0.5<\alpha(\mu)<4$ and $0.2<\alpha(\mu)<5$, respectively - see Table 2 and Fig. 6 . The results are also consistent the hypothesis that $\alpha(\mu)$ does not vary significantly with $\mu$ for the 0.05 and 0.01 significance levels.

In the energy range of $\gamma_{1}$ no conclusions about anisotropy can be drawn because high values of the K-S probabilities for the distribution of $\gamma_{1}$ do not permit us to distinguish them, though, the results are not inconsistent with the albedo model which does not predict significant variation of $\gamma_{1}$ with $\mu$ and $\alpha$ in this energy range - see Fig. 4.

Finally, the small number of suitable events at high energy prevented us from analysing $\gamma_{2}$. On the basis of the albedo model, see Fig. 4, we do not expect to find a significant influence of the anisotropy in the $\gamma_{2}$ energy range, but this prediction is not tested by the presented data.

\section{Low-energy cutoff in $\bar{F}(E)$ demanded by the data}

The survey presented in the this paper is also suitable for analysis of flares where the hard X-ray spectrum may indicate suspicious features in $\bar{F}(E)$.

Using a regularised inversion technique (Kontar et al. 2004; Scullion et al. in preparation), we determined the mean electron flux distribution $\bar{F}(E)$ from count spectra of several events
Fig. 5. Distributions of $\gamma_{0}$ for "centre" $(\mu>0.9$, dashed line) and "limb" $(\mu<0.1$, solid line $)$ flares. The left plot corresponds to observed $\gamma_{0}$, the right plot is for spectra corrected for isotropic albedo, i.e. with directivity $\alpha=1$.

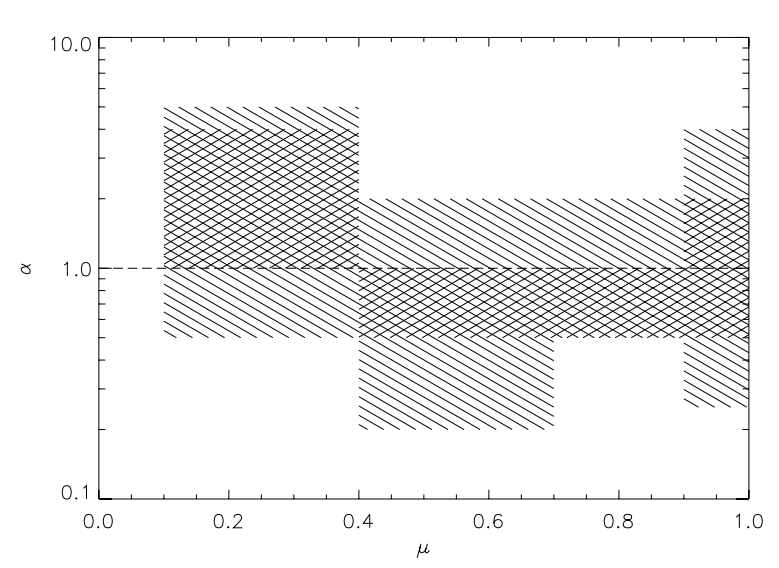

Fig. 6. Directivity at different $\mu$ determined from the distributions of $\gamma_{0}$ at the limb and a given range of $\mu$. Cross-hatched areas show the directivity for which the hypothesis that the observed distribution at a given range of $\mu$, and the distribution $I_{\mathrm{M}}$ at the limb with added albedo contribution, are drawn from the same parent distribution cannot be rejected at the 0.05 significance level; crossed areas correspond to the 0.01 significance level. See also Table 2. The dashed line shows the isotropic case, i.e. $\alpha(\mu)=1$.

which had either flat photon spectra or a large change in local spectral index with energy. Such spectral behaviour is expected for photon spectra either affected by the albedo - see Fig. 1 or produced by $\bar{F}(E)$ with an absence of electrons in same energy range. We found several new cases which exhibit a statistically significant dip in $\bar{F}(E)-$ e.g. Fig. 7 (left). The positions and spectral indices of events with such a dip are indicated in Fig. 4. However, when the isotropic albedo correction was applied (i.e. $\alpha(\mu)=1$ ), all such dips in $\bar{F}(E)$ were removed e.g. Fig. 7 (right). Our set of events includes the Sep. 17, 2002 (Kontar et al. 2006) and Apr. 25, 2002 flares (Kontar \& Brown 2006a) for which a statistically significant dip in $\bar{F}(E)$ at energies below $30 \mathrm{keV}$ has been reported, neglecting albedo. 

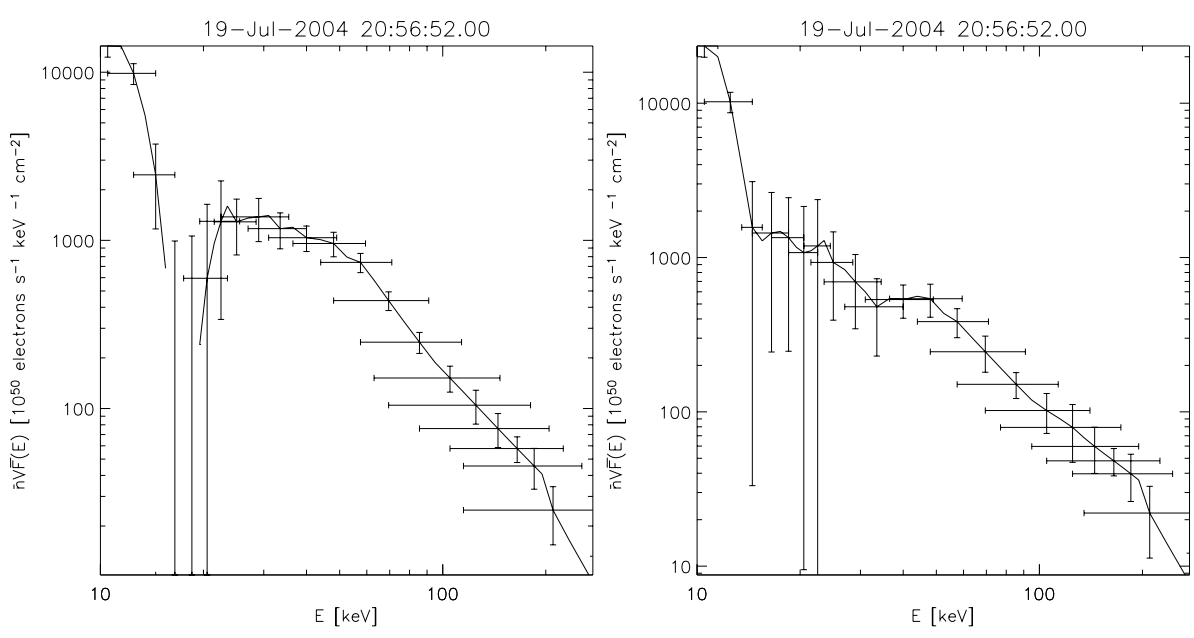

Fig. 7. $\bar{F}(E)$ corresponding to the total (left) and primary (right) flare photon spectrum for an event close to the disc centre (19 Jul. 2004). Vertical error bars correspond to $3 \sigma$ uncertainties, horizontal lines show the FWHM of energy resolution determined from regularised inversion.
The albedo contribution in events close to the limb should be negligible. Therefore, features in $\bar{F}(E)$ for events there could not be ascribed to the distortion of the photon spectra by albedo. $\bar{F}(E)$ was determined for a number of events close to the limb which showed flattening of the photon spectra. None of these events close to the solar limb $(\mu<0.5)$ was found to have a statistically significant dip in $\bar{F}(E)$, but revealed only some flattening of $\bar{F}(E)$. All events with determined $\bar{F}(E)$ are indicated in Fig. 4.

\section{Discussion and conclusions}

The high energy resolution of RHESSI down to $\sim 1 \mathrm{keV}$ and a large database have allowed us to study statistically 398 events not affected by pulse pile-up but having sufficient count rate to measure local spectral indices in three different energy bands. Combining these with flare position measurements we analysed centre-to-limb variation of hard X-ray flare spectra. The data clearly show a distinct variation of low energy spectral indices with heliocentric angle. This dependency is strong for spectral index in the range 15-20 keV but very weak for higher energies. The mean values of low energy index $\gamma_{0}$ are decreasing for larger $\mu$ (smaller heliocentric angles). We have compared centre-to-limb spectral variations with the predictions of albedo induced spectral index change (Kontar et al. 2006) for primary spectra assumed to be power-law and found the behaviour of mean spectral indexes $\bar{\gamma}_{0}, \bar{\gamma}_{1}$, and $\bar{\gamma}_{2}$ (energy ranges $15-20 \mathrm{keV}$, 20-35 keV, and 35-50 keV) to be consistent with the spectral change due to Compton backscattering albedo. For $\gamma_{1}$ and $\gamma_{2}$ at higher energies there is very little constraint information in the data.

The intensity of backscattered photons is determined by intensity of downward beamed photons, so we can estimate the ratio of downward/observer directed photons. Although our data set contains large number of events of photon spectra with high energy resolution, it can be only used to test the hard X-ray anisotropy below $20 \mathrm{keV}$. Even for an anisotropic primary source, the albedo induced spectral change and centre-tolimb variation are significant only below $20 \mathrm{keV}$ - see Figs. 1 and 4 (which applies for a primary power-law photon spectrum). The inferred anisotropy ratio, $0.2<\alpha(\mu)<5$, (Fig. 6) in the 15-20 keV range, and especially its large spread, allows no clear conclusion about the anisotropy. Moreover, at these energies anisotropy of electron beam emission can be masked by isotropic thermal emission, thus making the determination of anisotropy from spatially integrated spectra difficult.
The value of anisotropy ratio obtained is consistent with the predictions of hard X-ray emission of downward collimated beam and cannot reject even quite strongly beamed cases. For example, the detailed model of Leach \& Petrosian (1983, Fig. 4) predicts $\alpha \lesssim 3$ at $22 \mathrm{keV}$ for disc centre events. On the other hand, the determined anisotropy $\alpha(\mu)$ is also consistent with isotropic X-ray emission and lends support to quite isotropic electron distribution when put together with the Kontar \& Brown (2006b) analysis of detailed spectra of individual events. The anisotropy parameter $\alpha(\mu)$ was also found not to need significant dependence on $\mu$ - see Fig. 6 - indicating that the beam $\mathrm{X}$-ray emission need not strongly vary in the upward hemisphere (different values of $\mu$ probe different viewing angles of upward emission). We stress that with $\alpha(\mu)$ we measure the anisotropy of downward directed photons rather than upward directed photons at different angles as was done in most previous studies of solar flare directivities.

The data analysed in the paper, as well as previous published results (Nitta et al. 1990; Fárník et al. 1997; Kašparová et al. 2005; Kontar \& Brown 2006a) describe events with observed photon spectra which are flat at low energies. Such behaviour suggests a low-energy cutoff in the mean electron spectrum as derived from such observed photon spectra. We show that all such flares were located close to the solar disc centre $\left(\mu>0.5\left(\theta<60^{\circ}\right)\right)$, where the albedo component must be properly taken into account. Nitta et al. (1990) reported that two flares observed with SMM on 15 October 1981 at 4:43 UT and on 29 March 1980 at 9:18 UT had $\gamma \sim 2$ and $\gamma=1.9$, respectively, both flares being located not far from disc centre $(\mu \geq 0.6)$. Fárník et al. (1997) observed a few flares with very flat observed hard X-ray photon spectra from the same active region near the disc centre $(\mu=1.0)$. We note that Zhang \& Huang (2003, 2004) studied the combined effect of the low-energy cutoff and albedo contribution to explain very flat photon spectra observed by Yohkoh/HXT and concluded that both effect could cause flattening of photon spectra. However, to account for albedo they used integral reflectivity (angle-averaged) which is not applicable to centre-to-limb studies.

We have applied an isotropic albedo correction to the flares in our survey and have found this makes the alleged low-energy cutoff (or a "dip") in electron spectra statistically insignificant. This survey strongly suggests the hypothesis that the low-energy cutoff inferred in the mean electron flux distribution is an artefact of the photospheric albedo. Hence, we can confidently confirm our earlier conclusion (Kontar et al. 2006) that low energy cutoff is a feature connected with albedo and not a physical 
property of the mean electron distribution. This result can substantially change the estimated flux and total number of electrons accelerated in a flare. Therefore, we strongly suggest that the albedo component must be considered in determination of electron beam properties from spatially integrated solar hard Xray spectra.

Acknowledgements. We are grateful to H. S. Hudson and C. M. Johns-Krull for their valuable comments. J.K. acknowledges the financial support of a Royal Society Incoming Short Visit grant and a PPARC RA post to visit University of Glasgow in 2005 and 2006, and of grants 205/04/0358 and 205/06/P135 of the Grant Agency of the Czech Republic, and of research plan AV0Z10030501 of the Astronomical Institute AS CR. E.P.K. and J.C.B. acknowledge the financial support of a PPARC Advanced Fellowship and Rolling Grant respectively. This work was also supported by the Visiting Science Program at International Space Science Institute, Bern, Switzerland.

\section{References}

Alexander, R. C., \& Brown, J. C. 2002, Sol. Phys., 210, 407

Alexander, R. C., \& Brown, J. C. 2006, in preparation

Bai, T., \& Ramaty, R. 1978, ApJ, 219, 705

Bogovalov, S. V., Kotov, Y. D., Zenchenko, V. M., et al. 1985, Sov. Astron. Lett., 11,322

Brown, J. C. 1971, Sol. Phys., 18, 489

Brown, J. C. 1972, Sol. Phys., 26, 441

Brown, J. C., Emslie, A. G., \& Kontar, E. P. 2003, ApJ, 595, L115

Catalano, C. P., \& van Allen, J. A. 1973, ApJ, 185, 335

Conway, A. J., Brown, J. C., Eves, B. A. C., \& Kontar, E. 2003, A\&A, 407, 725

Datlowe, D. W., Elcan, M. J., \& Hudson, H. S. 1974, Sol. Phys., 39, 155

Datlowe, D. W., O’Dell, S. L., Peterson, L. E., \& Elcan, M. J. 1977, ApJ, 212, 561

Drake, J. F. 1971, Sol. Phys., 16, 152
Elwert, G., \& Haug, E. 1971, Sol. Phys., 20, 413

Fárník, F., Hudson, H., \& Watanabe, T. 1997, A\&A, 320, 620

Johns, C. M., \& Lin, R. P. 1992, Sol. Phys., 137, 121

Kane, S. R. 1974, in Coronal Disturbances, ed. G. A. Newkirk, IAU Symp., 57, 105

Kašparová, J., Karlický, M., Kontar, E. P., Schwartz, R. A., \& Dennis, B. R. 2005, Sol. Phys., 232, 63

Kontar, E. P., \& Brown, J. C. 2006a, Adv. Space Res., 38, 945

Kontar, E. P., \& Brown, J. C. 2006b, ApJ, 653, L149

Kontar, E. P., Piana, M., Massone, A. M., Emslie, A. G., \& Brown, J. C. 2004, Sol. Phys., 225, 293

Kontar, E. P., MacKinnon, A. L., Schwartz, R. A., \& Brown, J. C. 2006, A\&A, 446,1157

Langer, S. H., \& Petrosian, V. 1977, ApJ, 215, 666

Leach, J., \& Petrosian, V. 1983, ApJ, 269, 715

Lin, R. P., Dennis, B. R., Hurford, G. J., et al. 2002, Sol. Phys., 210, 3

Magdziarz, P., \& Zdziarski, A. A. 1995, MNRAS, 273, 837

McTiernan, J. M., \& Petrosian, V. 1991, ApJ, 379, 381

Nitta, N., Dennis, B. R., \& Kiplinger, A. L. 1990, ApJ, 353, 313

Ohki, K.-I. 1969, Sol. Phys., 7, 260

Petrosian, V. 1973, ApJ, 186, 291

Phillips, K. J. H. 1973, The Observatory, 93, 17

Pintér, Š. 1969, Sol. Phys., 8, 142

Press, W., Teukolsky, S., Vetterling, W., \& Flannery, B. 1992, Numerical recepies in C: the art of scientific computing, 2nd edn. (Cambridge University Press) Roy, J.-R., \& Datlowe, D. W. 1975, Sol. Phys., 40, 165

Schwartz, R. A., Csillaghy, A., Tolbert, A. K., et al. 2002, Sol. Phys., 210, 165

Scullion, E., Kontar, E. P., \& Kašparová, J., in preparation

Smith, D. M., Lin, R. P., Turin, P., et al. 2002, Sol. Phys., 210, 33

Tomblin, F. F. 1972, ApJ, 171, 377

Vestrand, W. T., Forrest, D. J., Chupp, E. L., Rieger, E., \& Share, G. H. 1987, ApJ, 322, 1010

Zhang, J., \& Huang, G. L. 2003, ApJ, 592, L49

Zhang, J., \& Huang, G. L. 2004, Sol. Phys., 219, 135 